\title{
Calculating the Cosmological Constant, and a Minimal Time Step, for Our Present Universe. In Fidelity to the Topics Spoken as a Presenter in the Zeldovich 4 Conference, September 11, 2020
}

\author{
Andrew Walcott Beckwith \\ Physics Department, College of Physics, Chongqing University, Chongqing, China \\ Email: Rwill9955b@gmail.com, abeckwith@uh.edu
}

How to cite this paper: Beckwith, A.W. (2021) Calculating the Cosmological Constant, and a Minimal Time Step, for Our Present Universe. In Fidelity to the Topics Spoken as a Presenter in the Zeldovich 4 Conference, September 11, 2020. Journal of High Energy Physics, Gravitation and Cosmology, 7, 403-415.

https://doi.org/10.4236/jhepgc.2021.72023

Received: December 28, 2020

Accepted: March 28, 2021

Published: March 31, 2021

Copyright $\odot 2021$ by author(s) and Scientific Research Publishing Inc. This work is licensed under the Creative Commons Attribution International License (CC BY 4.0).

http://creativecommons.org/licenses/by/4.0/

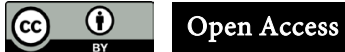

\begin{abstract}
The following is a rendition of what was presented by the author, September 11, 2020 in the DE section of that conference. The topics, while not original, are in strict fidelity with the topics the author was allowed to present in ICRANET Zeldovich 4, 2020. We present a history of the evolution of the cosmological constant "issue" starting with its introduction by Einstein for a static universe, which did not work out because his static universe solution to the Ricci Scalar problem, and GR was and is UNSTABLE. Another model of the cosmological constant has a radius of the Universe specified which is proportional to one over the square root of the cosmological constant, whereas our idea is to use the matching of two spacetime first integrals, for isolating a nonperturbative cosmological constant solution right at the surface of the start of expansion of the universe, i.e. a phenomenological solution to the cosmological constant involves scaling of a radius of the PRESENT universe. Our presented idea is to instead solve the Cosmological constant at the surface of the initial space-time bubble, using the initially derived time step, delta $t$, as input for the Cosmological constant. As it is, the Zeldovich 4 Section I was in was for Dark Energy, so in solving the initial value of the Cosmological constant, I am giving backing to one of the models of DE as to why the Universe reaccelerates one billion years ago. We conclude as to a reference to a multiverse generalization of Penrose Cyclic Conformal Cosmology as input into the initial nonsingular space-time bubble.
\end{abstract}


Keywords

Minimum Scale Factor, Cosmological Constant, Space-Time Bubble, CCC Cosmology (Penrose)

\section{Introduction, Using a Nonsingular Start to the Universe}

Our initial start is to use the ideas given in a JHEPC publication [1] and we replicate the beginning of that document to come up with a minimum time step. We will then use this starting point, for minimum time to come up with what is one of the simplest DE models which exist, i.e. the one for which there is an unvarying in the present era Cosmological Constant. The idea is as follows. We give an argument as to the existence of a minimum time step, at the surface of a space-time bubble. Then we discuss afterwards the failure of the initial Einstein solution for a static universe, which involves a nonstable solution which is for a very LARGE radius for an allegedly "unchanging" universe. That model, abandoned has been in part replaced by the cosmological constant as proportional to one over the square of the radius of an "observed" (?) universe which is in part unsatisfactory because it assumes perforce that we know the boundaries of an expanding universe (no we do not) which will then lead up to our model of comparing two first integrals at the surface of space-time to come up with an unvarying over time cosmological constant parameter. From then on we refer to the Karen Freeze Dark Stars [2] for formation of super-massive black holes to eat up Dark mater (increasing percentage of DE), and Abhay Ashtekar treatment of how a non-singular starts to the universe (as he did it) contributed to a taming of inhomogeneities as given by Figure 9.11, page 257 of Dodelson and Schmidt [3]. Ashtekar [4] claimed that the Nonsingular bubble is not decisive, but I disagree for reasons I will discuss in the manuscript. An elementary discussion of how a fixed cosmological constant contributes to acceleration of expansion concludes our manuscript. With suggested tests and caveats and concerns brought up in a conclusion, the multiverse idea I generalized from CCC cosmology [1] plays a role. And research follow ups.

\section{First of All the Minimum Time Step Issue Which We Will Find Is Important}

In [5] pages 212-213, we have that there is a Minkowski simple model for massive gravity, leading to

$$
m \cdot \partial_{t}\left(a^{2}-a^{3}\right)=0
$$

whereas if we us [6], and [1] and really look at [7], we can add in the following as to the scale factor used in Equation (1) which uses at the surface of a presumed non singular start to the expansion of the universe

$$
a(t)=a_{\min } t^{\gamma}
$$


Leading to [7] the inflaton.

$$
\phi=\sqrt{\frac{\gamma}{4 \pi G}} \ln \left\{\sqrt{\frac{8 \pi G V_{0}}{\gamma(3 \gamma-1)}} \cdot t\right\}
$$

Equation (1) and Equation (2) lead us to the interesting restraint of what we refer to as a minimum time step, below

$$
t=\left(\frac{2}{3 a_{\min }}\right)^{1 / \gamma}
$$

Our preliminary consideration is to have the time, in Equation (4) commensurate with Planck time, whereas a radius of the "bubble" of initial space-time commensurate with Planck length, using the dimensions given in [8].

Why is this initial minimum time step so important? We will see that answered as to the formation of the solution to the cosmological constant problem. But first a review of the unstable Einstein solution for a "static universe".

\section{Why Is the above about a Minimum Time Step Important? Underpins Nonsingular Start to the Universe}

Enter in a discussion of the failure of the static universe model (Einstein), plus the fault of having the radius of the universe, squared proportional to one over the Cosmological Constant. We will then go to the Klauder procedure.

First the Einstein "static universe". To do this, we go to page 144 of [9] by Plebasnki, and Krasinski. Here is their solution. Using the Ricci scalar, $R$, this is what they got for Einstein the value of the Ricci scalar so that

$$
R(\text { Ricci scalar })=g^{u v} R_{u v}=R_{E}=\frac{1}{\sqrt{-\Lambda}}=\frac{c}{2 \sqrt{\pi G \rho}}
$$

The density is, here, $\rho$, would have to be absurdly low, whereas $\Lambda$ has to be $10^{-50} / \mathrm{cm}^{2}$ as given by pp $410-411$ of Misner, Thorne, and Wheeler, [10]. And the fault of this is that by an analysis of this given on page 275 , of Plebasnki and Krasinski [9], that any perturbation of $R$ (Ricci scalar $)=g^{u v} R_{u v}=R_{E}$ would lead to either lead to expanding or contracting universe states.

\section{So What Do We Have Left? In Phenomenology, the Idea of a Linkage to the Radius of the Universe for $\Lambda$}

In Wesson, [11], pages 192-3, we have that there could be, if $L=$ Radius of unicerse, the value

$$
\Lambda \equiv 3 / L^{2}
$$

The question we then have to ask is then, what IS the radius of the universe? Is the universe a sphere?" What shape?

\section{Now for the General Relativity First Integral. From [1]}

We use the Padmanabhan $1^{\text {st }}$ integral [7] of the form, with the third entry of Equation (1) having a Ricci scalar defined via [9] and usually the curvature $\aleph$ set 
as extremely small, with the general relativity [1]

$$
\begin{aligned}
& S_{1}=\frac{1}{2 \kappa} \int(\Re-2 \Lambda) \cdot \sqrt{-g} \mathrm{~d}^{4} x \\
& g=\operatorname{det} g_{u v} \\
& \Re=6 \cdot\left(\left(\frac{\ddot{a}}{a}\right)+\left(\frac{\dot{a}}{a}\right)^{2}+\frac{\aleph}{a^{2}}\right)
\end{aligned}
$$

Also, the variation of $\delta g_{t t}=a_{\min }^{2} \phi$ as given by [1] will have an inflaton, $\phi$ given by [1] Leading to [1], [7] to the inflaton which is combined into other procedures for a solution to the cosmological constant problem. Here, $a_{\min }$ is a minimum value of the scale factor [1].

\section{Next for the Idea from Klauder}

We are going to go to page 78 by Klauder [1], [12] of what he calls on page 78 a restricted Quantum action principle which he writes as: $S_{2}$ where we write a 1-1 equivalence as in [1] [12]

$$
S_{2}=\int_{0}^{T} \mathrm{~d} t \cdot\left[p(t) \dot{q}(t)-H_{N}(p(t), \dot{q}(t))\right] \approx S_{1}=\frac{1}{2 \kappa} \int(\Re-2 \Lambda) \cdot \sqrt{-g} \mathrm{~d}^{4} x
$$

Our assumption is that $\Lambda$ is a constant, hence we assume then the following approximation, from [1]

$$
\begin{aligned}
& \frac{p_{0}^{2}}{2}=\frac{p_{0}^{2}(\tilde{N})}{2}+\tilde{N} \text {; for } 0<\tilde{N} \leq \infty \text {; and } q=q_{0} \pm p_{0} t \\
& V_{N}(x)=0 \text {; for } 0<x \leq 1 \\
& V_{N}(x)=\tilde{N} \text {; otherwise } \\
& H_{N}(p(t), \dot{q}(t))=\frac{p_{0}^{2}}{2}+\frac{(\hbar \cdot \pi)^{2}}{2}+\tilde{N} \text {; for } 0<\tilde{N} \leq \infty
\end{aligned}
$$

Our innovation is to then equate $q=q_{0} \pm p_{0} t$ and to assume small time step values. Then [1]

$$
\Lambda \approx \frac{-\left[\frac{V_{0}}{3 \gamma-1}+2 \tilde{N}+\frac{\gamma(3 \gamma-1)}{8 \pi G t^{2}}\right]_{t=t_{p}}}{\frac{1}{\kappa} \cdot \int \sqrt{-g} \mathrm{~d}^{3} x}+6 \cdot\left(\left(\frac{\ddot{a}}{a}\right)+\left(\frac{\dot{a}}{a}\right)^{2}+\frac{\aleph=0}{a^{2}}\right)_{t=t_{p}}
$$

\section{Now We Have a Candidate Balance of Contributions to $\Lambda$ at the Start of the Universe. Assuming DE Is $\Lambda$ How Do We Account for a Possible Reduction of the Role of DM? From the Early Universe?}

According to Freese, Rindler-Daller Spolyar and Valluri massive BH of a mass $10^{8}$ solar masses formed early on, in the center of spiral galaxies, forming from DM stars as presented, here [2]. The point is this, that the following pie chart probably had more DM than today, with the Dark Stars perhaps shrinking the amount of DM and boosting the total budget of DR in the cosmos (Figure 1). 


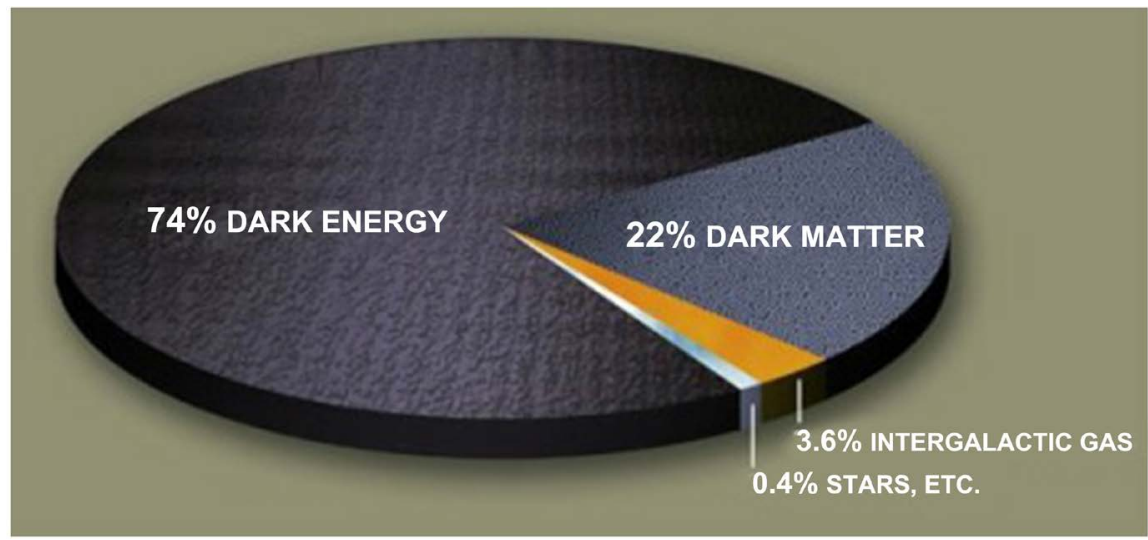

Figure 1. Attributed to commonly available descriptions [13].

We do not exactly know what the $\mathrm{DE}$ is, but here is a clue as to its role in the present universe. And DM may have been considerably more prevalent than today, if the SM black holes from the center of galaxies are from giant DM stars.

\section{A Review of Nonsingular Cosmologies and the Friedman Equation, and How We Utilize Equation (4)}

A given in our work is that within the nucleation of space-time, that time, even as given in Equation (5) simply does not exist, but that we will be able to use the results of Freeze [14] as far as a nonsingular Friedman equation to get

$$
H^{2}=\frac{8 \pi}{3 M_{P}^{2}} \cdot\left(\rho-\frac{\rho^{2}}{2|\sigma|}\right)
$$

Here, we have that $\rho$ is a space-time density function, whereas $\sigma$ is related to the tension of a space-time bubble presumably of the order of a Planck radius. And we are also using what is given in [13] as far as a Dark Energy model, which we write, for energy density, as given by, if $\mathfrak{R}$ is the Ricci scalar [10]. We have also the discussion given in [13] and set $\kappa$ as spacetime curvature, and so then we have

$$
\rho_{D E}=\frac{3 \tilde{\alpha}}{8 \pi} \cdot\left(\dot{H}+2 H^{2}+\frac{\kappa}{a^{2}}\right)=-\frac{\tilde{\alpha}}{8 \pi} \cdot \Re
$$

In terms of the bubble of spacetime before inflation, we submit that time does not really exist and that then we will be considering a rewrite of the above as having, effectively $\dot{H}=0$. And the term $\rho_{D E}=\rho$. And if we apply Equation (11) we have within the bubble of spacetime this value for $\rho_{D E}=\rho$ at just about the surface of a bubble

$$
\rho^{2}-\rho \cdot(2|\sigma|)\left(1-\left(\frac{3 \tilde{\alpha}}{8 \pi}\right)^{-1} \cdot\left(\frac{16 \pi}{3 M_{P}^{2}}\right)^{-1}\right)-\left(\frac{16 \pi}{3 M_{P}^{2}}\right)^{-1} \cdot(2|\sigma|) \cdot \frac{\kappa}{a^{2}}=0
$$

Unlike the Wheeler De Witt formulation where there is a zero net "energy" value commensurate with no time evolution of the wave function of the universe, as seen in [15], with variants proposed in [16], we will be involved in using an 
input into the initial physical system for reasons brought up in [1], and this will allow us then to say something about the formulation of a time step at the surface of the space-time bubble, according to the minimum uncertainty principle, i.e. at the surface of the bubble, of space time, say of about one Planck length in radius, we would have then a minimum timestep defined by using [17] $\Delta E \Delta t \equiv \hbar$. If so then, if the initial volume is of the cube of a Planck length, we have a time step defined via, if the $\tilde{\alpha}$ value, were small, we would be probably be looking at

$$
\Delta t=\frac{l_{\text {Planck }}^{3} \hbar /|\sigma|}{\left(1-\left(\frac{3 \tilde{\alpha}}{8 \pi}\right)^{-1} \cdot\left(\frac{16 \pi}{3 M_{P}^{2}}\right)^{-1}\right) \pm \sqrt{\left(\left(1-\left(\frac{3 \tilde{\alpha}}{8 \pi}\right)^{-1} \cdot\left(\frac{16 \pi}{3 M_{P}^{2}}\right)^{-1}\right)\right)^{2}+\left(\frac{16 \pi}{3 M_{P}^{2}}\right)^{-1} \cdot(2|\sigma|)^{-1} \cdot \frac{8 \kappa}{a^{2}}}}
$$

A limiting solution, of all things turns out to be if the curvature term is small, with, among other approximations, to be, surprise, to have the bubble tension term dropping out. But this is not a GENERAL solution

$$
\Delta t \approx 2 l_{\text {Planck }}^{3} \hbar \cdot \frac{\left(\left(\frac{3 \tilde{\alpha}}{8 \pi}\right)^{-1}-\left(\frac{16 \pi}{3 M_{P}^{2}}\right)\right)}{\frac{8 \kappa}{a^{2}}}
$$

In either case, we will be doing our calculations to determine what this has to say as to the frequency of a signal from this event, as well as the strength of GW, and then also the possible polarization states. This would have to be contrasted with Equation (4), as to what that says about bounding values for the input into Equation (14). Needless to state the value of time picked, either Equation (4) and/or Equation (12) or giving equality between Equation (4) and Equation (14) would be a way to approximate the first values of the terms as far as time evolution of the scale factors given in Equation (10) above.

\section{Now Looking at What Was Discussed by Abhay Ashtekar in Zeldovich 4. On September 7, 2020 [4]}

In our Figure 2, we copy what was done by Ashtekar, in Zelsovich 4 as to what was part of anisotropic fits to the $\mathrm{E}$ and $\mathrm{B}$ polarization, as given Ashtekar, in [4] referred to a method as to smoothing out the error bars as given via use of LQG with the Starobinsky Potential given as

$$
V(\phi)=\frac{3 M^{2}}{32 \pi} \cdot\left(1-\mathrm{e}^{-\sqrt{\frac{16 \pi G}{3} \phi^{2}}}\right)
$$

what we are suggesting is that the nonsingular start to the Universe, not just the LQG may play a role in terms of resolving anisotropic conditions as given in Figure 2, in low 1 value contingencies. But this issue, brought up in Figure 2 has been an endless source of comments for me in several years of Rencontres De Moriond Cosmology seminars in La Thuile, Italy. 


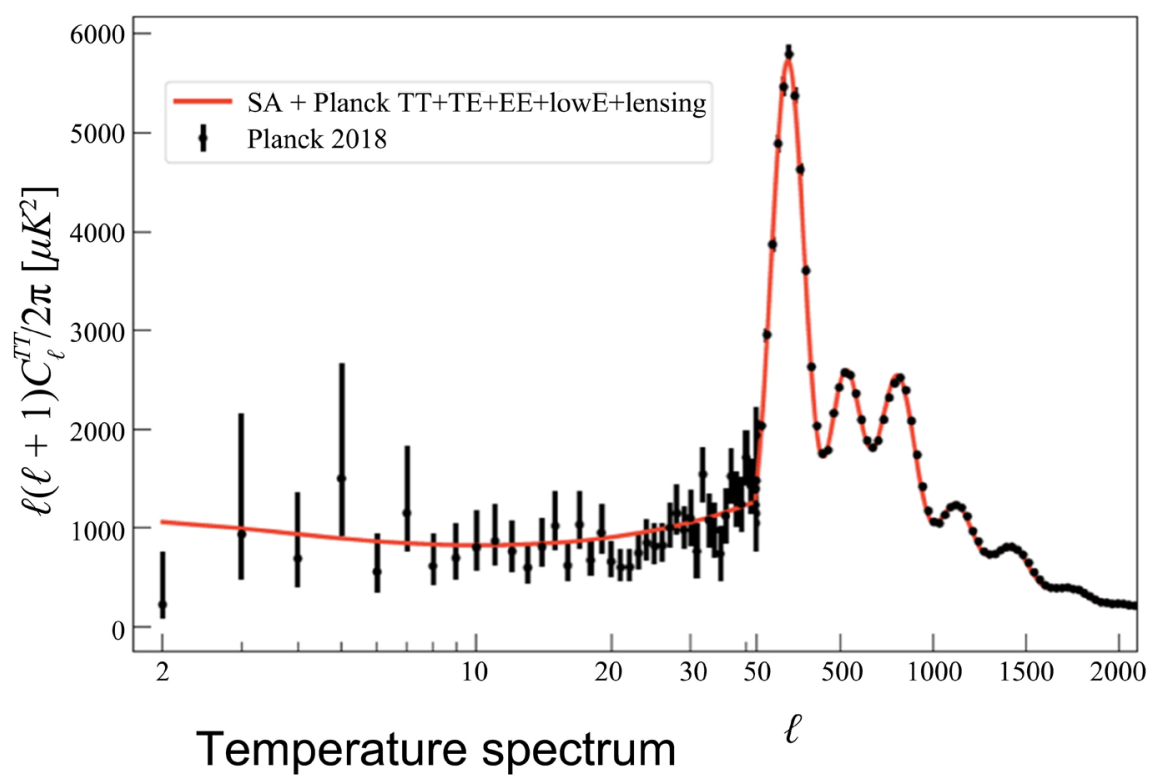

Figure 2. Abhay Ashtekar in Zeldovich 4. On September 7, 2020 [4]. The crust of what he presented is his claim that by Loop Quantum gravity, could solve CMBR data glitches [4].

\section{Next, Referring to the Generalization of the Penrose CCC Cosmology, for Consistency of Cosmological Parameters and Input as to Our Nonsingular Start Leading to Equation (18)}

We are extending Penrose's suggestion of cyclic universes [18], black hole evaporation, and the embedding structure our universe is contained within, this multiverse embeds BHs and may resolve what appears to be an impossible dichotomy. The following is largely taken from [1] and has serious relevance to the final part of the conclusion. That there are no fewer than $N$ universes undergoing Penrose "infinite expansion" (Penrose) [18] contained in a mega universe structure. Furthermore, each of the $N$ universes has black hole evaporation, with the Hawking radiation from decaying black holes. If each of the $N$ universes is defined by a partition function, called, $\left\{\Xi_{i}\right\}_{i=N}^{i=1}$ then there exists an information ensemble of mixed minimum information correlated as about $10^{7}-10^{8}$ bits of information per partition function in the set $\left.\left\{\Xi_{i}\right\}_{i=N}^{i=1}\right|_{\text {before }}$, so minimum information is conserved between a set of partition functions per universe [1]

$$
\left.\left\{\Xi_{i}\right\}_{i=N}^{i=1}\right|_{\text {before }}=\left.\left\{\Xi_{i}\right\}_{i=N}^{i=1}\right|_{\text {after }}
$$

However, there is non-uniqueness of information put into each partition function. Furthermore Hawking radiation from the black holes is collated via a strange attractor collection in the mega universe structure to form a new big bang for each of the $N$ universes represented by $\left\{\Xi_{i}\right\}_{i=N}^{i=1}$. Verification of this mega structure compression and expansion of information with a non-uniqueness of information placed in each of the $N$ universes favors ergodic mixing treatments of initial values for each of $N$ universes expanding from a singularity be- 
ginning. The $n_{f}$ value, will be using ( $\left.\mathrm{Ng}, 2008\right) S_{\text {entropy }} \approx n_{f}$. [19]. How to tie in this energy expression, as in Equation (17) will be to look at the formation of a nontrivial gravitational measure as a new big bang for each of the $N$ universes as by $n\left(E_{i}\right)$ the density of states at a given energy $E_{i}$ for partition function, [20].

$$
\left\{\Xi_{i}\right\}_{i=N}^{i=1} \approx\left\{\int_{0}^{\infty} \mathrm{d} E_{i} n\left(E_{i}\right) \mathrm{e}^{-E_{i}}\right\}_{i=1}^{i=N}
$$

Each of $E_{i}$ identified with Equation (17) above, are with the iteration for $N$ universes [1] [18] (Penrose, 2006). Then the following holds, by asserting the following claim to the universe, as a mixed state, with black holes playing a major part, which is using Ergodic mixing, to a degree [1] [21]

\section{Claim 1}

$\left.\left.\frac{1}{N} \sum_{j=1}^{N} \Xi_{j}\right|_{\text {before nucleation(present universe) }} \underset{\text { nucleation(present universe) }}{\longrightarrow} \Xi_{i}\right|_{\text {after nucleation(present universe) }}$

For $N$ number of universes, with each $\left.\Xi_{j}\right|_{\text {before nucleation(present universe) }}$ for $j=1$ to $N$ being the partition function of each universe just before the blend into the RHS of Equation (16) above for our present universe. Also, each of the independent universes given by are constructed by the absorption of one to ten million black holes taking in energy, i.e. (Penrose) [1]. Furthermore, the main point is similar to what was done in [1] reproduced below in terms of general ergodic mixing [21]

Claim 2

$$
\left.\Xi_{j}\right|_{\text {before nucleation(present universe) }} \approx \sum_{i=1}^{\text {maximum }} \Xi_{i}^{\text {(black holes) }}
$$

what is done in Claim 1 and Claim 2 is to come up as to how a multi dimensional representation of black hole physics enables continual mixing of spacetime; [1] [21] largely as a way to avoid the Anthropic principle, as to a preferred set of initial conditions. Claim 2 is to use what is known as CCC cosmology, which can be thought of as the following. First. Have a big bang (initial expansion) for the universe. FOR SAY redshift $Z=10$, a ten billion years ago, SMBH formation starts. Matter-energy is vacuumed up by the SMBHs [1].

Then the main methodology in the Penrose proposal has been in Equation (20) evaluating a change in the metric $g_{a b}$ by a conformal mapping $\hat{\Omega}$ to [1] giving space for which $\lambda_{D E} \approx 10^{30} \ell_{\text {Planck }}$, And here we have an inversion of the metric according to the rules given in Equation (20) and Equation (21) as follows

$$
g_{a b}(\text { modified })=\hat{\Omega} g_{a b}
$$

Penrose's suggestion has been to utilize the following [1]

$$
\hat{\Omega} \underset{\mathrm{ccc}}{\longrightarrow} \hat{\Omega}^{-1}
$$

In fall into cosmic black hopes has been the main mechanism which the author asserts would be useful for the recycling apparent in Equation (21) above 
with the caveat that the invariance of the Planck $\hbar$ combined with Equation (21) above gives a good indication of a uniform mass to a graviton, per cycle, as far as heavy gravity, provided that $\hbar_{\text {old cycle(ccc) }}=\hbar_{\text {new cycle(ccc) }}$ holds' [1].

\section{One Big Takeaway from All This, Is That the Cosmological Constant Is Rooted Right into the Value of a Massive Graviton}

I.e. by this amount by Novello, [22]

$$
m_{g}=\frac{\hbar \sqrt{\Lambda}}{c}
$$

\section{Where This Leaves Us}

1) First of all, we have that Equation (22) leaves a solid link as to the interlinkages between massive gravitons and the calculated cosmological constant.

2) To be confirmed, do we have evidence of a multi universe contribution as to the formation of an initial universe expansion? This needs to be confirmed experimentally.

3) As outlined, this can lead to confirming or denying the truth of what Corda raised in his article about a minimal alteration of GR, which is recorded in [23], [24].

4) Our methodology if confirmed and worked on, will lead to among other things. A Non perturbative, but different procedure as to bounding the cosmological constant, and by extension DE. Since DE is, in its simplest iteration the cosmological constant, we have that in terms of the transition from the interior bubble of the space time start to inflation to traditional space time inflation the following to explore, with $d S$ the surface of an event horizon between feeding into the spacetime bubble, and cosmic inflation.

$$
\begin{aligned}
& c \rho_{d S}=\left.\frac{\left(R_{00}-g_{00} R\right)}{8 \pi}\right|_{d S} \\
& \left.\left.\Leftrightarrow \frac{\left(-3 \frac{\ddot{a}}{a}-g_{00} 6 \Lambda\right)}{8 \pi}\right|_{d s} \approx \frac{\left(-3 \frac{\ddot{a}}{a}+g_{00} 6\left(\frac{\ddot{a}}{a}+\left(\frac{\dot{a}}{a}\right)^{2}+\frac{2 \kappa}{a^{2}}\right)\right)}{8 \pi}\right|_{d s}
\end{aligned}
$$

5) See this final take away as to what the cosmological constant is equivalent to.

6) In order to obtain maximum results, we will be stating that the following will be assumed to be equivalent. [1], i.e. the term comes from using expression of [25] [26]

$$
\sqrt{g_{t t}} \cdot\left(\frac{g_{t t} V_{3}^{2}}{V_{3}}+k_{2} V_{3}^{1 / 3}\right) \approx \frac{1}{2 \kappa} \cdot \int \sqrt{-g} \mathrm{~d}^{3} x \cdot\left[6 \cdot\left(\left(\frac{\ddot{a}}{a}\right)+\left(\frac{\dot{a}}{a}\right)^{2}+\frac{\aleph=0}{a^{2}}\right)_{t=t_{p}}\right]
$$




$$
\sqrt{g_{t t}} \lambda V_{3} \approx \frac{1}{2 \kappa} \cdot \int \sqrt{-g} \mathrm{~d}^{3} x \cdot(2 \Lambda)
$$

leading to

$$
\lambda(\text { Lagrangian multiplier }) \approx \frac{1}{\kappa} \cdot \sqrt{\frac{-g}{\delta g_{t t}}} \cdot \Lambda
$$

\section{Conclusions, Relevance to Black Hole Production}

Our assumption is that the Lagrangian multiplier is roughly equivalent to a mass which is about $10^{4}$ times the mass of a Planck sized black hole mass, i.e. that we have Black holes initially produced which are of say $10^{2}$ times the Planck mass. In Corda's recent work [27], we have that $n$ is the quantum number $n$ put in where Planck mass is normalized to 1 , so then, if there are $10^{2}$ black holes of mass $M=10^{2} M_{\text {planck }} \equiv 10^{2}$. Here we make the following simplification of a so-called Horizon volume Equation (20) to read as. After normalizing Planck mass as being $=1$, then by [28] we state

$$
\left.\Delta V_{n-1, n}\right|_{\text {total }} \approx 10^{2} \times\left.\Delta V_{n-1, n}\right|_{\text {per black hole }} \approx 10^{2} \times 16 \pi \cdot\left(\frac{n}{4 \times 10^{4}}\right) \cdot\left|\sqrt{1-\left(\frac{n-1}{n}\right)}\right|
$$

Our supposition is that there are $10^{2}$ mini black holes, and a mass of $10^{2}$ times Planck mass, per each black hole, so that we find $n$ for quantum number. So if we assume [28] conditions. Then we have an entropy count, on r.h.s. as

$$
16 \pi \cdot\left(\frac{n}{4 \times 10^{4}}\right) \cdot\left|\sqrt{1-\left(\frac{n-1}{n}\right)}\right| \approx 10^{6}
$$

i.e. that say 1000 times Planck length, we have the beginning of say creation of 100 mini black holes, each of mass about 100 times Planck mass which would put a huge restriction upon the admissible quantum value, $n$.

Final comment are as to why I published this talk as given in Zeldovich 4. See the following.

All these topics were presented up to a point in the Zeldovich 4 conference, as can be seen here [28] and that essential proofing of this main idea, the comparison of the two first integrals as a way to obtain the cosmological constant near the initial phases of expansion of the universe is seen in [29], which was not reviewed by the Russian Journal who did not take the idea of my presentation, already peer reviewed in [29] seriously. In addition, the Russian Journal which allegedly is taking selected articles of the Zeldovich 4 conference, did not note either [30], or [31] or [31], which in their abstracts gave convincing proof of nonsingular initial configuration cosmologies seriously. Further proof of the existence of nonsingular cosmologies can be seen in [31], as well as in the Ettore Majoranja International science series as shown in [32] and [33].

Finally the author wishes to point out the existence of [32] by J. M. Khalantnikov, called Qualitative cosmology. What evidently was not appreciated as to the gist of the nonsingular start to my problem, is in some sense not materially 
different from what was done in [32] as far as conservative Hamiltonian systems.

All these entries will make their way into future publications and I wish to thank JHEPGC for a venue to display the gist of what I tried to convey on line in Zeldovitch 4, on September 11, 2020 as a talk in the Dark Energy section of that conference.

\section{Acknowledgements}

This work is supported in part by National Nature Science Foundation of China grant No. 11375279.

\section{Conflicts of Interest}

The author declares no conflicts of interest regarding the publication of this paper.

\section{References}

[1] Beckwith, A. (2020) How to Obtain a Mass of a Graviton, and Does This Methodology Lead to Voids? Journal of High Energy Physics, Gravitation and Cosmology, 6, 416-439. https://doi.org/10.4236/jhepgc.2020.63032

[2] Freese, K., Rindler-Daller, T., Spolyar, D. and Valluri, M. (2016) Dark Stars: A Review. Reports on Progress in Physics, 79, Article ID: 066902. https://doi.org/10.1088/0034-4885/79/6/066902 https://iopscience.iop.org/article/10.1088/0034-4885/79/6/066902

[3] Dodelson, S. and Schmidt, F. (2020) Modern Cosmology. 2nd edition, Elsevier Press, Cambridge.

[4] Ashtekar, A. (2020) Quantum Gravity in the Sky? Alleviating Tensions in the CMB Using Planck Scale Physics. http://www.icranet.org/images/stories/Meetings/ZM4/presentations/Ashtekar.pdf

[5] Tolley, A.J. (2015) Cosmological Applications of Massive Gravity. In: Papantonopoulos, E., Ed., Modifications of Einstein's Theory of Gravity at Large Distances, Vol. 892, Springer Verlag, Cham, 203-224. https://doi.org/10.1007/978-3-319-10070-8 8

[6] D’Amico, G., de Rham, C., Dubosvsky, S., Gabadadze, G., Pirtskhalava, D. and Tolley, A.J. (2011) Massive Cosmologies. Physical Review D, 84, Article ID: 124046. https://arxiv.org/abs/1108.5231 https://doi.org/10.1103/PhysRevD.84.124046

[7] Thanu, P. (2006) An Invitation to Astrophysics. World Scientific Series in Astronomy and Astrophysics, Vol. 8, World Scientific, Singapore.

[8] Wesson, P.S. (1980) The Application of Dimensional Analysis to Cosmology. Space Science Reviews, 27, 109-153. https://doi.org/10.1007/BF00212237

[9] Jerzy, P. and Andrzej, K. (2012) An Introduction to General Relativity and Cosmology. 1st Edition, Cambridge University Press, New York City.

[10] Misner, C., Thorne, K. and Wheeler, J. (2017) Gravitation. Princeton University Press, Princeton.

[11] Wesson, P. and Overduin, J. (2019) Principles of Space-Time-Matter, Cosmology, Particles, and Waves in Five Dimensions. World Scientific, Singapore.

[12] Klauder, J. (2015) Enhanced Quantization: Particles, Fields \& Gravity. World Scien- 
tific Press, Hackensack. https://doi.org/10.1142/9452

[13] Li, M., Li, X.-D., Wang, S. and Wang, Y. (2015) Dark Energy. Peking University-World Scientific Advanced Physics Series Vol. 1, World Scientific, Singapore. https://doi.org/10.1142/9293

[14] Freeze, K., Brown, M. and Kinney, W. (2012) The Phantom Bounce, A New Proposal for an Oscillating Cosmology. In: Mercini-Houghton, L. and Vaas, R., Eds., The Arrows of Time: A Debate in Cosmology, Vol. 172, Springer Verlag, Berlin, Heidelberg, 149-156. https://doi.org/10.1007/978-3-642-23259-6 7

[15] Carlo, R. (2001) Notes for a Brief History of Quantum Gravity. 9th Marcel Grossmann Meeting, Roma, July 2000, arXiv: gr-qc/000606

[16] Kolb, E. and Turner, M. (1990) The Early Universe (Frontiers in Physics). Addison and Westley, California.

[17] Griffiths, D.J. (2004) Introduction to Quantum Mechanics. 2nd Edition, Prentice Hall, Upper Saddle River.

[18] Penrose, R. (2006) Before the Big Bang: An Outrageous New Perspective and Its Implications for Particle Physics. Proceedings of 10 th European Conference EPAC 2006, Edinburgh, 26-30 June 2006, 2759-2762.

[19] Ng, Y.J. (2008) Spacetime Foam: From Entropy and Holography to Infinite Statistics and Nonlocality. Entropy, 10, 441-461. https://doi.org/10.3390/e10040441

[20] Poplawski, N. (2011) Cosmological Constant from Quarks and Torsion. Annalen der Physik, 523, 291-295. https://doi.org/10.1002/andp.201000162

[21] Dye, H. (1965) On the Ergodic Mixing Theorem. Transactions of the American Mathematical Society, 118, 123-130.

https://doi.org/10.1090/S0002-9947-1965-0174705-8

http://www.ams.org/journals/tran/1965-118-00/S0002-9947-1965-0174705-8/S00029947-1965-0174705-8.pdf

[22] Novello, M. (2005) The Mass of the Graviton and the Cosmological Constant Puzzle. https://arxiv.org/abs/astro-ph/0504505

[23] Corda, C. (2009) Interferometric Detection of Gravitational Waves: The Definitive Test for General Relativity. International Journal of Modern Physics D, 18, 2275-2282. https://doi.org/10.1142/S0218271809015904 https://arxiv.org/abs/0905.2502

[24] Corda, C. (2008) Primordial Production of Massive relic Gravitational Waves from a Weak Modification of General Relativity. Astroparticle Physics, 30, 209-215. https://doi.org/10.1016/j.astropartphys.2008.09.003 https://arxiv.org/abs/0812.0483

[25] Hamber, H.W. and Williams, R.M. (2011) Discrete Wheeler-DeWitt Equation. Physical Review D, 84, Article ID: 104033. arXiv:1109.2530. https://doi.org/10.1103/PhysRevD.84.104033

[26] Hamber, H.W., Toriumi, R. and Williams, R.M. (2012) Wheeler-DeWitt Equation in 2+1 Dimensions. Physical Review D, 86, Article ID: 084010. arXiv: 1207.3759. https://doi.org/10.1103/PhysRevD.86.084010

[27] Feleppa, F., Licata, I. and Corda, C. (2019) Hartle-Hawking Boundary Conditions as Nucleation by de Sitter Vacuum. Physics of the Dark Universe, 26, Article ID: 199381. https://doi.org/10.1016/j.dark.2019.100381 https://arxiv.org/abs/1909.07824

[28] Beckwith, A. (2018) Using "Enhanced Quantization" to Bound the Cosmological Constant, and Computing Quantum Number n for Production of 100 Relic Mini 
Black Holes in a Spherical Region of Emergent Space-Time. Journal of High Energy Physics, Gravitation and Cosmology, 4, 549-566.

https://doi.org/10.4236/jhepgc.2018.43033

http://www.icranet.org/images/stories/Meetings/ZM4/presentations/Beckwith.pdf

[29] Beckwith, A. (2021) Using "Enhanced Quantization" to Bound the Cosmological Constant, (for a Bound-on Graviton Mass), by Comparing Two Action Integrals (One Being from General Relativity) at the Start of Inflation. In: Sidharth, B.G., Murillo, J.C., Michelini, M. and Perea, C., Eds., Fundamental Physics and Physics Education Research, Springer Nature, Geneva, 21-35.

https://doi.org/10.1007/978-3-030-52923-9 3

[30] Parker, L. and Wang, Y. (1990) Avoidance of Singularities in Relativity through Two-Body Interactions. Physical Review D, 42, 1877-1883.

https://doi.org/10.1103/PhysRevD.42.1877

[31] Parker, L. (1991) Avoidance of Gravitational Singularities through Two Body Interactions. Annals of the New York Academy of Sciences, 631, 31-39. https://doi.org/10.1111/j.1749-6632.1991.tb52628.x

[32] Khalantnikov, I.M. (1991) Qualitative Cosmology. In: Zichichi, A., de Sabbata, V., and Sanchez, N., Eds., Gravitation and Modern Cosmology: The Cosmological Constant Problem, Springer, Boston, 65-85.

https://doi.org/10.1007/978-1-4899-0620-5 8

[33] Rosen, N. (1991) A Simple Model of the Universe without Singularities. In: Zichichi, A., de Sabbata, V., and Sanchez, N., Eds., Gravitation and Modern Cosmology, the Cosmological Constant Problem, Springer, Boston, 151-156.

https://doi.org/10.1007/978-1-4899-0620-5 14 